\title{
Degree spectra and immunity properties
}

\author{
Barbara F. Csima ${ }^{1, *}$ and Iskander S. Kalimullin ${ }^{2, * *}$ \\ ${ }^{1}$ Department of Pure Mathematics \\ University of Waterloo \\ Waterloo, ON, Canada N2L 3G1 \\ URL:www.math.uwaterloo.ca/ csima \\ E-mail adress: csima@math.uwaterloo.ca \\ ${ }^{2}$ Chebotarev Research Institute of Mathematics and Mechanics \\ Kazan State University \\ 420008, Kazan, Russia \\ E-mail adress: Iskander.Kalimullin@ksu.ru
}

Key words Degree spectra of algebraic structures, immune and hyperimmune sets and degrees MSC (2000) 03C57,03D28, 03D45

Copyright line will be provided by the publisher

We analyze the degree spectra of structures in which different types of immunity conditions are encoded. In particular, we give an example of a structure whose degree spectrum coincides with the hyperimmune degrees. As a corollary, this shows the existence of an almost computable structure the complement of the degree spectrum of which is uncountable.

Copyright line will be provided by the publisher

\section{Introduction}

Let $\mathcal{N}$ be a countable algebraic structure in a finite language $\Sigma$ (where the equality symbol $=$ belongs to $\Sigma$ ) whose universe is a subset of $\omega$. We denote via $\mathcal{D}(\mathcal{N})$ the set of all atomic sentences and their negations which are true in $\mathcal{N}$ (here we use some computable numbering of the sentences in the language $\Sigma^{*}=\Sigma \cup \omega$ to identify $\mathcal{D}(\mathcal{N})$ with a subset of $\omega$ ). The degree spectrum of a countable structure $\mathcal{M}$ is the set of all Turing degrees of sets $\mathcal{D}(\mathcal{N})$ for any $\mathcal{N} \cong \mathcal{M}$ with universe $\omega$ :

$$
\operatorname{Sp}(\mathcal{M})=\{\operatorname{deg}(\mathcal{D}(\mathcal{N})): \mathcal{M} \cong \mathcal{N} \& \operatorname{dom}(\mathcal{N})=\omega\}
$$

A structure $\mathcal{M}$ is computable if the zero degree, $\mathbf{0}$, belongs to $\operatorname{Sp}(\mathcal{M})$. By the following result the degree spectrum of a computable structure is either $\{\mathbf{0}\}$, or the class $\mathbf{D}$ of all Turing degrees.

Theorem 1.1 (Knight [13]). Let $\mathcal{A}$ be a countable structure in a finite language. Then exactly one of following statements holds:

1. if $\mathbf{c} \in \mathbf{S p}(\mathcal{A})$ and $\mathbf{c} \leq \mathbf{d}$, then $\mathbf{d} \in \mathbf{S p}(\mathcal{A})$;

2. $\mathbf{S p}(\mathcal{A})=\{\mathbf{0}\}$ (such structures are called trivial).

We will say that a nontrivial countable algebraic structure $\mathcal{M}$ is almost computable, if the measure of the degree spectrum $\operatorname{Sp}(\mathcal{M})$ is equal to one or, equivalently, the measure of the class

$$
\mathcal{C}(\mathcal{M})=\left\{X \in 2^{\omega}: \operatorname{deg}(X) \in \mathbf{S p}(\mathcal{M})\right\}
$$

is one under the standard uniform measure on the Cantor space $2^{\omega}$. Note that the last class is the countable union of the classes

$$
C_{\Phi}(\mathcal{M})=\left\{X \in 2^{\omega}: \Phi^{X} \text { is the atomic diagram of a copy of } \mathcal{M}\right\}
$$

\footnotetext{
* B. Csima was partially supported by Canadian NSERC Discovery Grant 312501.

** I. Kalimullin was partially supported by RFBR Grant 05-01-00605.
} 
where $\Phi$ is any Turing operator. By the Kolmogorov $0-1$ Law a structure is almost computable if and only if the measure of at least one $C_{\Phi}(\mathcal{M})$ is nonzero.

The first examples of almost computable structures which are not computable were constructed by Slaman [17], and independently, by Wehner [20]. Namely, they found structures $\mathcal{M}$ such that $\mathbf{S p}(\mathcal{M})=\mathbf{D}-\{\mathbf{0}\}$, or equivalently,

$$
\mathcal{C}(\mathcal{M})=\left\{X \in 2^{\omega}: X \text { is not computable }\right\}
$$

It follows from the paper [4] that for any computable ordinal $\alpha$ there is a structure with the degree spectrum $\left\{\mathbf{x} \in \mathbf{D} \mid \Delta_{\alpha+1}^{0}(\mathbf{x}) \nsubseteq \Delta_{\alpha+1}^{0}\right\}$. In particular, there are almost computable structures without arithmetical copies. For arithmetical structures, it is known that there are structures whose degree spectra have the form $\{\mathbf{x} \in \mathbf{D} \mid \mathbf{x} \not \mathbf{a}\}$, where $\mathbf{a}$ is any low [10] or computably enumerable [11] degree. In particular, there are arithmetical almost computable structures without $\Delta_{2}^{0}$ copies. Note also, that by [12] there is a degree $\mathbf{a} \leq \mathbf{0}^{\prime \prime}$ such that the collection $\{\mathbf{x} \in \mathbf{D} \mid \mathbf{x} \not \mathbf{a}\}$ is not the degree spectrum of a structure.

Note that all structures mentioned above are almost computable because the set $2^{\omega}-\mathcal{C}(\mathcal{M})$ is countable. In this paper we will give examples of almost computable structures $\mathcal{M}$ for which the set $2^{\omega}-\mathcal{C}(\mathcal{M})$ is uncountable. Moreover, we will construct a structure $\mathcal{G}$ such that $\operatorname{Sp}(\mathcal{G})$ coincides with the hyperimmune degrees.

We now recall the definitions of various immunity properties.

Definition 1.2 1. An infinite set $X$ is immune if it has no infinite computable subset.

2. An infinite set $X$ is hyperimmune if there is no computable function $f$ such that $\left\{D_{f(n)}\right\}_{n \in \omega}$ is a disjoint strong array and for all $n \in \omega, D_{f(n)} \cap X \neq \emptyset$.

3. An infinite set $X$ is hyperhyperimmune if there is no computable function $f$ such that $\left\{W_{f(n)}\right\}_{n \in \omega}$ is a disjoint weak array and for all $n \in \omega, W_{f(n)} \cap X \neq \emptyset$.

Here $\left\{D_{n}\right\}_{n \in \omega}$ is a computable list of the finite sets, and $\left\{W_{n}\right\}_{n \in \omega}$ is a uniform enumeration of the c.e. sets. By a disjoint strong array $\left\{D_{f(n)}\right\}_{n \in \omega}$ we mean a sequence of disjoint sets given by a computable function $f$. By a disjoint weak array $\left\{W_{f(n)}\right\}_{n \in \omega}$ we mean a sequence of disjoint finite sets given by a computable function $f$.

We have a very nice characterization of the hyperimmune sets. Recall that for $X=\left\{x_{0}<x_{1}<x_{2}<\cdots\right\}$, the principal function for $X$ is defined by $p_{X}(n)=x_{n}$. We say the function $f$ dominates the function $g$ if for all but finitely many $x, f(x)>g(x)$.

Theorem 1.3 (Kuznecov, Medvedev [15], Uspenskii [19]) An infinite set $X$ is hyperimmune if and only if no computable function dominates $p_{X}$.

We say a Turing degree $\mathbf{d}$ is immune (hyperimmune, hyperhyperimmune) if it contains an immune (hyperimmune, hyperhyperimmune) set.

The immune degrees are exactly the noncomputable degrees (see [1]). The characterization of hyperimmune sets passes to degrees, in the sense that a degree $\mathbf{d}$ is hyperimmune if and only if there exists a $\mathbf{d}$-computable function that is not dominated by any computable function.

A general theorem of Jockusch [8] shows that the immune, hyperimmune, and hyperhyperimmune degrees are all upward closed in the Turing degrees. The proof makes strong use of the fact that any infinite subset of an immune (hyperimmune, hyperhyperimmune) set is also immune (hyperimmune, hyperhyperimmune).

An infinite set $X$ is bi-immune (bi-hyperimmune, bi-hyperhyperimmune) if both it and its complement are immune (hyperimmune, hyperhyperimmune). A Turing degree is said to have any of these properties if it contains a set with the corresponding property.

It is known that all hyperimmune degrees are bi-hyperimmune (see e.g. Kurtz [14]). Jockusch [6] showed the existence of a degree $\mathbf{d}$ that is immune but not bi-immune. Also, the bi-hyperhyperimmune degrees are properly contained in the hyperhyperimmune degrees. This was communicated to the authors by Jockusch, who pointed out that since all $\Delta_{2}^{0}$ hyperhyperimmune sets are in fact strongly hyperhyperimmune, and since bistrongly hyperhyperimmune sets do not exist, there can be no $\Delta_{2}^{0}$ bi-hyperhyperimmune sets. On another hand, it is known that there are $\Delta_{2}^{0}$ hyperhyperimmune sets, for example comaximal sets. Thus, the containment is proper. 
Note that an infinite subset of a bi-immune set need not be bi-immune, so Jockusch's general theorem does not give upward closure of the bi-immune degrees. However, in [7] Jockusch gave a non-uniform proof that the bi-immune degrees are upward closed in the Turing degrees. Since the bi-hyperimmune degrees coincide with the hyperimmune degrees, they are upward closed in the Turing degrees. It is not known to us whether the bi-hyperhyperimmune degrees are upward closed in the Turing degrees.

Since the degree classes of bi-immune, hyperimmune, and hyperhyperimmune degrees are all upward closed, it is natural to ask whether they can be realized as the degree spectra of a structure. In this paper, we will succeed for the class of the hyperimmune degrees, and suggest some structures the degree spectra of which in one case might be the bi-immune degrees and in another case might be the bi-hyperhyperimmune degrees.

\section{Notation}

We follow standard notation for Computability Theory, as found in Cooper [1] and Soare [18]. For a survey of Computable Model Theory, we recommend Harizanov [5].

Definition 2.1 For a set $F \subseteq \omega$ and $n \in \omega$, we let $\{n\} \oplus F$ denote the following infinite graph. It is an $\omega$-chain (edges between $m$ and $m+1$ ), with an $n+5$-cycle linked to 0 , and a 3-cycle linked to $m$ if $m \in F$ and a 4 -cycle linked to $m$ if $m \notin F$.

Definition 2.2 To each $\sigma \in 2^{<\omega}$ and $n \in \omega$, we let $\{n\} \oplus \sigma$ denote the following graph. It is a $l h(\sigma)$-chain, with an $n+5$-cycle linked to 0 , and a 3-cycle linked to $m$ if $\sigma(m)=1$ and a 4-cycle linked to $m$ if $\sigma(m)=0$.

Definition 2.3 For any $e \in \omega$ and any set $X \subseteq \omega$, let $X^{[e]}=\{x \mid\langle e, x\rangle \in X\}$.

Definition 2.4 For any $e \in \omega$ and any set $X \subseteq \omega$, let $X+e=\{x+e \mid x \in X\}$.

Notation 2.5 For $\sigma \in 2^{<\omega}$, we let $l h(\sigma)$ denote the length of $\sigma$, i.e., $\operatorname{lh}(\sigma)=\mu n\{n \notin \operatorname{dom}(\sigma)\}$.

To each $\sigma \in 2^{<\omega}$ we associate the finite set $F_{\sigma}=\{n \mid \sigma(n)=1\}$.

\section{Noncomputable}

We begin by recounting an example of Wehner, of a structure with degree spectrum exactly the noncomputable Turing degrees.

For Wehner's example, let $\mathcal{J}=\left\{\{n\} \oplus F|n \in \omega \wedge| F \mid<\infty \wedge F \neq W_{n}\right\}$. That is, let $\mathcal{J}$ be the disjoint union of one copy each of graphs of the form $\{n\} \oplus F$ where $F$ is finite and $F \neq W_{n}$.

Wehner actually used a more universal coding, including infinitely many copies of each component of the form $\{n\} \oplus F$, but for our purposes it is enough to use exactly one of each. Note also that this leads to a rigid structure.

Before showing that this structure has the desired degree spectrum, we begin on a theme and re-characterize the degrees that compute $\mathcal{J}$.

Lemma 3.1 There is an $X$-computable copy of $\mathcal{J}$ if and only if there exists a set $Y \equiv_{T} X$ such that $(\forall e)\left[\left|Y^{[e]}\right|<\infty\right]$ and $(\forall e)\left[Y^{[e]} \neq W_{e}\right]$.

Pro of. Suppose we have an $X$-computable copy of $\mathcal{J}$. We build $Y \equiv_{T} X$ with the required property. To code $X$ into $Y$ we will ensure that $e \in X$ iff $0 \in Y^{[e]}$. We $X$-computably search through the copy of $\mathcal{J}$ until we find an $e+5$ cycle that corresponds to a component of the form $\{e\} \oplus F$ where $0 \in F$ iff $e \in X$. There are finite sets that disagree with $W_{e}$ that contain 0 and ones that do not contain 0 , so we will certainly find such a component. We let $Y^{[e]}=F$.

Now suppose $Y$ is a set such that $(\forall e)\left[\left|Y^{[e]}\right|<\infty\right]$ and $(\forall e)\left[Y^{[e]} \neq W_{e}\right]$. We describe how to build a $Y$-computable copy of $\mathcal{J}$ by stages as follows.

Stage 0: $\mathcal{J}_{0}$ is empty.

Stage $s+1$ : For uncommitted $\{n\} \oplus \sigma \in \mathcal{J}_{s}$, if for all $x \in \operatorname{dom}(\sigma)$ we have $x \in W_{n, s+1} \Longleftrightarrow \sigma(x)=1$, then compute $k$ such that $W_{k}+s=W_{n}-\{0, \ldots, s\}$, and make the commitment to extend $\{n\} \oplus \sigma$ to $\{n\} \oplus \widehat{\sigma_{\chi}} \chi_{Y[k]}+s$. For all other uncommitted $\{n\} \oplus \sigma \in \mathcal{J}_{s}$, extend to $\{n\} \oplus \widehat{\sigma} 0$ in $\mathcal{J}_{s+1}$. For committed $\{n\} \oplus \sigma \in \mathcal{J}_{s}$, extend one place towards their commitment. For all $n \leq s+1$, for all $\sigma \in 2^{s+1}$, place a copy of $\{n\} \oplus \sigma$ into $\mathcal{J}_{s+1}$ if no such copy yet exists. 
This completes the construction.

Verification: It is easy to see by induction on $s$ that at the end of each stage $s$, all components in $\mathcal{J}_{s}$ have the form $\{n\} \oplus \sigma$, with $l h(\sigma)=s$, and that if $\{n\} \oplus \sigma \neq\{n\} \oplus \tau$ then $\sigma$ and $\tau$ are incomparable. Thus for each set $F$ and each $n \in \omega$, there can be at most one copy of $\{n\} \oplus F$ in $\mathcal{J}$.

Once a component of the form $\{n\} \oplus \sigma$ is introduced into $\cup_{s} \mathcal{J}_{s}$, it is either extended to $\{n\} \oplus \widehat{\sigma} 0^{\infty}$ or $\{n\} \oplus \widehat{\sigma 0^{m}} \chi_{Y[k]+s}$ for some $m, k$, and $s$. In either case the resulting component is of the form $\{n\} \oplus F$ where $|F|<\infty$.

If $F$ is finite and $F \neq W_{n}$ then there exists a stage $s$, an initial segment $\sigma \in 2^{<\omega}$, and a number $x$ such that $\chi_{F}=\widehat{\sigma} 0^{\infty}$, and such that for all $t \geq s, x \in \operatorname{dom}(\sigma) \wedge\left[\left(x \in W_{n, t} \wedge \sigma(x)=0\right) \vee\left(x \notin W_{n, t} \wedge \sigma(x)=1\right)\right]$. Thus $\{n\} \oplus F \in \cup_{s} \mathcal{J}_{s}$.

Now suppose $\{n\} \oplus F \in \cup_{s} \mathcal{J}_{s}$. We have already seen that $|F|<\infty$. It remains to show that $F \neq W_{n}$. Assume for a contradiction that $F=W_{n}$. Then there exists a stage $s$ and an initial segment $\sigma \subset \chi_{F}$ such that $\{n\} \oplus \sigma \in \mathcal{J}_{s}$ and $x \in W_{n, s+1} \Longleftrightarrow \sigma(x)=1$. At stage $s+1$ in the construction, we would have made the commitment to extend $\{n\} \oplus \sigma$ to $\{n\} \oplus \widehat{\chi_{\chi_{Y}[k]}+s}$ for $k$ such that $W_{k}+s=W_{n}-\{0, \ldots, s\}$. Now

$$
\begin{aligned}
W_{n}=F & \Rightarrow \chi_{W_{n}}=\widehat{\sigma \chi_{Y}^{[k]}+s} \\
& \Rightarrow W_{n}-\{0, \ldots, s\}=Y^{[k]}+s \\
& \Rightarrow W_{k}+s=Y^{[k]}+s \\
& \Rightarrow W_{k}=Y^{[k]}
\end{aligned}
$$

a contradiction.

Theorem 3.2 (Wehner) $\operatorname{Sp}(\mathcal{J})=\mathbf{D}-\{0\}$.

Proof. Suppose there is an $X$-computable copy of $\mathcal{J}$. Then there exists $Y \leq_{T} X$ such that $(\forall e)\left[\left|Y^{[e]}\right|<\infty\right]$ and $(\forall e)\left[Y^{[e]} \neq W_{e}\right]$. If $Y$ were computable, then we could define a computable function $g$ by $W_{g(x)}=Y^{[x]}$. By the Recursion Theorem, there exists $n$ such that $W_{n}=Y^{[n]}$, a contradiction. Hence $Y$ is not computable, and so neither is $X$.

Now suppose that $X$ is not computable. We will find $Y \leq_{T} X$ satisfying the conditions of Lemma 3.1 as follows ${ }^{1}$.

Since the degree of $X$ is immune, we may assume without loss of generality that $X$ is immune (for example, we can replace a noncomputable set $X$ by the immune set $X_{1}=\left\{\sigma \in 2^{<\omega} \mid \sigma \subset X\right\}$ ). For all $e \in \omega$ let

$$
Y^{[e]}=\left\{p_{X}(0)\right\} \cup\left\{p_{X}(n+1) \mid n \in \omega \wedge W_{e, n} \neq W_{e, n+1} \wedge W_{e, n+1} \subseteq X\right\}
$$

where $p_{X}$ is the principal function for $X$. Clearly $Y \leq_{T} X$ (since $p_{X} \leq_{T} X$ and $p_{X}(n) \geq n$ for all $n \in \omega$ ). Let $e \in \omega$ be given.

Suppose at first that $W_{e} \cap \bar{X} \neq \emptyset$. Then $Y^{[e]}$ is finite because there are only finitely many $n$ with $W_{e, n} \subseteq X$. Also $Y^{[e]} \neq W_{e}$ since $Y^{[e]} \subseteq X$ and $W_{e} \cap \bar{X} \neq \emptyset$.

Suppose now that $W_{e} \subseteq X$. Then $W_{e}$ is finite because $X$ is immune. It follows that $Y^{[e]}$ is finite because there are only finitely many $n$ with $W_{e, n} \neq W_{e, n+1}$. Let $m$ be the least integer such that $W_{e}=W_{e, n}$ for all $n \geq m$. Then $p_{X}(m) \in Y^{[e]}-W_{e}$ since $y \in W_{e, m}$ implies $y<m$ by the standard assumption on $W_{e, s}, s \in \omega$.

In both cases, $Y^{[e]}$ is finite and not equal to $W_{e}$.

\section{At least bi-immune, but not hyperimmune}

For our next example, let $\mathcal{F}=\left\{\{n\} \oplus F|n \in \omega \wedge| F \mid<\infty \wedge\left(\left|W_{n}\right|=\infty \rightarrow W_{n} \cap F \neq \emptyset\right)\right\}$.

Lemma 4.1 There exists an $X$-computable copy of $\mathcal{F}$ if and only if there exists $Y \equiv_{T} X$ such that $(\forall e)\left[\left|Y^{[e]}\right|<\right.$ $\infty]$ and $(\forall e)\left[\left|W_{e}\right|=\infty \rightarrow Y^{[e]} \cap W_{e} \neq \emptyset\right]$.

\footnotetext{
1 The authors are very grateful to the referee for simplifying this proof.
} 
Pro of. Suppose we have an $X$-computable copy of $\mathcal{F}$. To define $Y^{[e]}$, search for a component of the form $\{e\} \oplus F$ where $0 \in F$ iff $e \in X$, and let $Y^{[e]}=F$.

Conversely, suppose $Y \equiv_{T} X$ is such that $(\forall e)\left[\left|Y^{[e]}\right|<\infty\right]$ and $(\forall e)\left[\left|W_{e}\right|=\infty \rightarrow Y^{[e]} \cap W_{e} \neq \emptyset\right]$. We describe how to build a $Y$-computable copy of $\mathcal{F}$ by stages as follows.

Stage 0: $\mathcal{F}_{0}$ is empty.

Stage $s+1$ : For uncommitted $\{n\} \oplus \sigma \in \mathcal{F}_{s}$, if $F_{\sigma} \cap W_{n, s+1}=\emptyset$ and $W_{n, s+1} \neq W_{n, s}$, then compute $k$

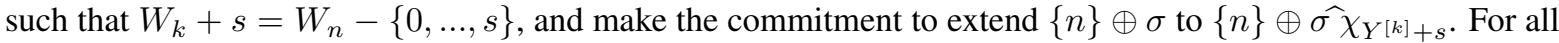
other uncommitted $\{n\} \oplus \sigma \in \mathcal{F}_{s}$, extend to $\{n\} \oplus \sigma^{\top} 0$ in $\mathcal{F}_{s+1}$. For committed $\{n\} \oplus \sigma \in \mathcal{F}_{s}$, extend one place towards their commitment. For all $n \leq s+1$, for all $\sigma \in 2^{s+1}$, place a copy of $\{n\} \oplus \sigma$ into $\mathcal{F}_{s+1}$ if no such copy yet exists.

This completes the construction.

Verification: It is easy to see by induction on $s$ that at the end of each stage $s$, all components in $\mathcal{F}_{s}$ have the form $\{n\} \oplus \sigma$, with $l h(\sigma)=s$, and that if $\{n\} \oplus \sigma \neq\{n\} \oplus \tau$ then $\sigma$ and $\tau$ are incomparable. Thus for each set $F$ and each $n \in \omega$, there can be at most one copy of $\{n\} \oplus F$ in $\mathcal{F}$.

Once a component of the form $\{n\} \oplus \sigma$ is introduced into $\cup_{s} \mathcal{F}_{s}$, it is either extended to $\{n\} \oplus \sigma^{-} 0^{\infty}$ or $\{n\} \oplus \widehat{\sigma^{0} 0^{m} \chi_{Y^{[k]}+s}}$ for some $m, k$, and $s$. In either case the resulting component is of the form $\{n\} \oplus F$ where $|F|<\infty$.

If $\left|W_{n}\right|<\infty$, then there is some stage $s$ after which $W_{n}$ gains no more members. Any $\{n\} \oplus \sigma$ introduced into $\cup_{s} \mathcal{F}_{s}$ after stage $s$ will be extended to $\{n\} \oplus \sigma^{-} 0^{\infty}$. Hence for every finite set $F$, a component of the form $\{n\} \oplus F$ will appear in $\cup_{s} \mathcal{F}_{s}$.

Similarly, if $F$ is finite and $W_{n} \cap F \neq \emptyset$, then there is a stage $s$ and a finite initial segment $\sigma \subset \chi_{F}$ such that $W_{n, t} \cap F_{\sigma \sim \sigma m} \neq \emptyset$ for all $m$ and all $t \geq s$, and hence there is a component of the form $\{n\} \oplus F$ in $\cup_{s} \mathcal{F}_{s}$.

Now suppose $\left|W_{n}\right|=\infty$ and $\{n\} \oplus F \in \cup_{s} \mathcal{F}_{s}$. There must have been some $\sigma \subset \chi_{F}$ such that $\{n\} \oplus \sigma$ was introduced into $\mathcal{F}$. Then at the next stage when $W_{n}$ gained a new member, if $F_{\sigma} \cap W_{n, s+1}=\emptyset$, we made

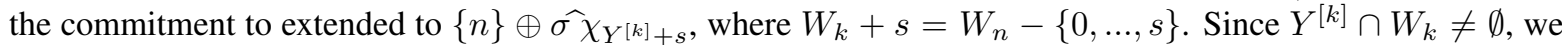
have $Y^{[k]}+s \cap W_{k}+s \neq \emptyset$. But since $\chi_{F}=\widehat{\sigma} \chi_{Y^{[k]}+s}$, we have $F \supseteq Y^{[k]}+s$. Also $W_{n}=W_{k}+s$. Hence $F \cap W_{n} \neq \emptyset$.

Theorem 4.2 If $X$ has hyperimmune degree, then there is an $X$-computable copy of $\mathcal{F}$.

Proof. Since $X$ is hyperimmune, there exists a function $g \leq_{T} X$ such that $g$ is not dominated by any computable function. That is, for any computable function $f,\left(\exists^{\infty} x\right)[f(x)<g(x)]$.

We construct $Y \leq_{T} X$ such that $(\forall e)\left[\left|Y^{[e]}\right|<\infty\right]$ and $(\forall e)\left[\left|W_{e}\right|=\infty \rightarrow Y^{[e]} \cap W_{e} \neq \emptyset\right]$, by finite extensions using $g$ as follows.

Let $\sigma_{s}^{[e]}$ denote the initial segment of the characteristic function of $Y^{[e]}$ that has been defined at the end of stage $s$.

Stage 0: Let $\sigma_{0}^{[e]}=\emptyset$.

Stage s+1: For all $e \leq s$, do as follows. If $F_{\sigma_{s}^{[e]}} \cap W_{e, s}=\emptyset$ and there is some $x>s$ with $x \in W_{e, g(s)}$, let $\sigma_{s+1}^{[e]} \supset \sigma_{s}^{[e]}$ be such that $x \in F_{\sigma_{s+1}^{[e]}}$. For all other $e \leq s$, let $\sigma_{s+1}^{[e]}=\sigma_{s}^{[e]} 0$.

This completes the construction.

Verification:

If $W_{e}$ is infinite then the function $\psi_{e}(s)=\mu t(\exists x>s)\left[x \in W_{e, t}\right]$ is total, and hence $\left(\exists^{\infty} s\right)\left[g(s)>\psi_{e}(s)\right]$. At the least stage $s$ such that $g(s)>\psi_{e}(s)$, the construction guarantees that $W_{e} \cap Y^{[e]} \neq \emptyset$.

Theorem 4.3 If there is an $X$-computable copy of $\mathcal{F}$ then $X$ has bi-immune degree.

Pro of. Suppose there is an $X$-computable copy of $\mathcal{F}$. Then there exists $Y \equiv_{T} X$ such that $(\forall e)\left[\left|Y^{[e]}\right|<\infty\right]$ and $(\forall e)\left[\left|W_{e}\right|=\infty \rightarrow Y^{[e]} \cap W_{e} \neq \emptyset\right]$. We will build a bi-immune set $Z \leq_{T} Y$. Since the bi-immune degrees are upward closed [7], this will show that $X$ has bi-immune degree.

We meet for all $e \in \omega$ the requirements:

$$
R_{e}:\left|W_{e}\right|=\infty \rightarrow W_{e} \cap Z \neq \emptyset
$$




$$
S_{e}:\left|W_{e}\right|=\infty \rightarrow W_{e} \cap \bar{Z} \neq \emptyset
$$

We construct $Z$ by stages as follows. At stage $s+1$, we decide whether or not $s \in Z$. In order to meet the requirement $R_{0}$, we will ensure that the finite set $Y^{[0]} \subseteq Z$. This will satisfy $R_{0}$ since if $W_{0}$ is infinite then $Y^{[0]} \cap W_{0} \neq \emptyset$. In order to satisfy $S_{0}$, we will similarly choose a finite set that is guaranteed to intersect $W_{0}$ if $W_{0}$ is infinite, and this time keep this finite set out of $Z$. Naturally, we cannot choose $Y^{[0]}$ to be this set, as (unless it is empty) it cannot be both a subset of $Z$ and $\bar{Z}$. In fact, we will continue with the plan to keep $Y^{[0]}$ out of $Z$ until we find the first member of $Y^{[0]}$. This is the moment when there is conflict between the desires of $R_{0}$ and $S_{0}$. Since $R_{0}$ has highest priority, we will choose a new set to keep out of $Z$ for the sake of $S_{0}$. Indeed, if $k$ is the greatest member of $Y^{[0]}$ that we've seen so far, then we will compute $n$ such that $W_{n}=W_{0}-\{0, \ldots, k\}$, and $S_{0}$ will desire to keep $Y^{[n]}$ out of $Z$. Since $Y^{[0]}$ is finite, $S_{0}$ will only have to change its set finitely often.

In the construction, we will make use of functions $f_{s}(e)$ and $g_{s}(e)$ that keep track of the witness sets for $R_{e}$ and $S_{e}$.

Stage 0: Let $f_{0}(e)=g_{0}(e)=e$ for all $e \in \omega$.

Stage $s+1$ : Choose the least $e \leq s$ such that $s \in Y^{\left[f_{s}(e)\right]} \vee s \in Y^{\left[g_{s}(e)\right]}$. If $s \in Y^{\left[f_{s}(e)\right]}$, then declare $s \in Z$; for $i>e$ set $W_{f_{s+1}(i)}=W_{i}-\{0, \ldots, s\}$; and for $i \geq e$ set $W_{g_{s+1}(i)}=W_{i}-\{0, \ldots, s\}$. If $s \in Y^{\left[g_{s}(e)\right]}$, then declare $s \notin Z$; for $i>e$ set $W_{f_{s+1}(i)}=W_{i}-\{0, \ldots, s\}$; and for $i>e$ set $W_{g_{s+1}(i)}=W_{i}-\{0, \ldots, s\}$. If no such $e$ exists, then declare $e \in Z$ and leave $f_{s+1}=f_{s}$ and $g_{s+1}=g_{s}$.

Verification: Since each $Y^{[k]}$ is finite, it follows by induction that $f(e)=\lim _{s} f_{s}(e)$ and $g(e)=\lim _{s} g_{s}(e)$ exist for all $e$. Moreover, for each $e, Y^{[f(e)]} \subseteq Z$ and $Y^{[g(e)]} \subseteq \bar{Z}$. Suppose $W_{e}$ is infinite. Since $W_{f(e)}=$ $W_{e}-\{0, \ldots, s\}$ for some $s, W_{f(e)}$ is also infinite. So $W_{f(e)} \cap Y^{[f(e)]} \neq \emptyset$. But since $W_{e} \supseteq W_{f(e)}$ and $Z \supset Y^{[f(e)]}$, we have $W_{e} \cap Z \neq \emptyset$. Similarly, $W_{e} \cap \bar{Z} \neq \emptyset$.

Corollary 4.4 The graph $\mathcal{F}$ is almost computable but the class of degrees $\mathbf{D}-\mathbf{S p}(\mathcal{F})$ is uncountable.

Proof. Martin (see [2]) showed that the measure of the members of hyperimmune degrees is equal to one. By Theorem $4.2, \mathcal{F}$ is almost computable.

Jockusch [6] proved the existence of nonzero bi-immune free degrees. His construction can be adapted to prove that there exist uncountably many bi-immune free degrees. Hence, Theorem 4.3 ensures that $\mathbf{D}-\mathbf{S p}(\mathcal{F})$ is also uncountable.

Note that this Theorem cannot be strengthened to state that any $X$ that can compute a copy of $\mathcal{F}$ must have hyperimmune degree. Indeed, it is possible to build a non-empty $\Pi_{1}^{0}$-class, all of whose members compute a copy of $\mathcal{F}$. But then, since every non-empty $\Pi_{1}^{0}$ class contains a member of hyperimmune-free degree [9], there is a hyperimmune-free degree computing a copy of $\mathcal{F}$.

Lemma 4.5 There is a non-empty $\Pi_{1}^{0}$ class, all of whose members compute a copy of $\mathcal{F}$.

Proof. Let $\psi$ be a selector function for $\left\{W_{e}\right\}_{e \in \omega}$. That is, let $\psi$ be a partial computable function such that for all $e \in \omega$ :

$$
W_{e} \neq \emptyset \Longleftrightarrow \psi(e) \text { is defined } \Longleftrightarrow \psi(e) \in W_{e} .
$$

Define

$$
C=\left\{X \subseteq \omega \mid(\forall e)\left[\left|X^{[e]}\right| \leq 1 \&\left(W_{e} \neq \emptyset \Longrightarrow \psi(e) \in X^{[e]}\right)\right]\right\}
$$

It is easy to see that $C$ forms a non-empty $\Pi_{1}^{0}$ class, and by Lemma 4.1, every $X \in C$ computes a copy of $\mathcal{F}$.

Question 4.6 Is $\mathbf{S p}(\mathcal{F})$ exactly the bi-immune degrees? 


\section{Hyperimmune}

Now consider

$$
\mathcal{G}=\left\{\{n\} \oplus F|n \in \omega \wedge| F \mid<\infty \wedge\left(\left\{D_{\varphi_{n}(m)}\right\}_{m \in \omega} \text { is a disjoint strong array } \rightarrow(\exists m)\left[D_{\varphi_{n}(m)} \subseteq F\right]\right)\right\}
$$

Lemma 5.1 There exists an $X$-computable copy of $\mathcal{G}$ if and only if there exists $Y \equiv_{T} X$ such that $(\forall e)\left[\left|Y^{[e]}\right|<\right.$ $\infty]$ and $(\forall e)\left[\left\{D_{\varphi_{e}(m)}\right\}_{m \in \omega}\right.$ is a disjoint strong array $\left.\rightarrow(\exists m)\left[D_{\varphi_{e}(m)} \subseteq Y^{[e]}\right]\right]$.

Proof. Suppose we have an $X$-computable copy of $\mathcal{G}$. To define $Y^{[e]}$, search for a component of the form $\{e\} \oplus F$ with $e \in X$ iff $0 \in F$, and let $Y^{[e]}=F$.

Conversely, suppose $Y \equiv_{T} X$ is such that $(\forall e)\left[\left|Y^{[e]}\right|<\infty\right]$ and $(\forall e)\left[\left\{D_{\varphi_{e}(m)}\right\}_{m \in \omega}\right.$ is a disjoint strong array $\left.\rightarrow(\exists m)\left[D_{\varphi_{e}(m)} \subseteq Y^{[e]}\right]\right]$. We describe how to build a $Y$-computable copy of $\mathcal{G}$ by stages as follows.

Stage 0: $\mathcal{G}_{0}$ is empty.

Stage $s+1$ : For uncommitted $\{n\} \oplus \sigma \in \mathcal{G}_{s}$, if $\varphi_{n, s+1}(m) \downarrow$ for some $m$ on which $\varphi_{n, s}(m) \uparrow$ and if $D_{\varphi_{n}(p)} \cap$ $D_{\varphi_{n}(q)}=\emptyset$ for all $p \neq q$ on which $\varphi_{n, s+1}(p) \downarrow$ and $\varphi_{n, s+1}(q) \downarrow$, and if $\neg(\exists m)\left[D_{\varphi_{e, s+1}(m) \downarrow} \subseteq F_{\sigma}\right]$, then do as follows. We can certainly compute $k$ such that if $\varphi_{n}$ is total then so is $\varphi_{k}$ and $\left\{\varphi_{k}(x)\right\}_{x \in \omega}=\left\{\varphi_{n}(x) \mid D_{\varphi_{n}(x)} \cap\right.$ $\{0, \ldots, s\}=\emptyset\}_{x \in \omega}$. We then make the commitment to extend $\{n\} \oplus \sigma$ to $\{n\} \oplus \chi_{\left(Y^{[k]}-\{0, \ldots, s\}\right) \cup F_{\sigma}}$. For all other uncommitted $\{n\} \oplus \sigma \in \mathcal{G}_{s}$, extend to $\{n\} \oplus \widehat{\sigma 0}$ in $\mathcal{G}_{s+1}$. For all $n \leq s+1$, for all $\sigma \in 2^{s+1}$, place a copy of $\{n\} \oplus \sigma$ into $\mathcal{G}_{s+1}$ if no such copy yet exists.

This completes the construction.

Verification: It is easy to see by induction on $s$ that at the end of each stage $s$, all components in $\mathcal{G}_{s}$ have the form $\{n\} \oplus \sigma$, with $l h(\sigma)=s$, and that if $\{n\} \oplus \sigma \neq\{n\} \oplus \tau$ then $\sigma$ and $\tau$ are incomparable. Thus for each set $F$ and each $n \in \omega$, there can be at most one copy of $\{n\} \oplus F$ in $\mathcal{G}$.

Since each $Y^{[k]}$ is finite, it is clear that all components of $\cup_{s} \mathcal{G}_{s}$ have the form $\{n\} \oplus F$ where $|F|<\infty$.

If $\varphi_{n}$ does not code a disjoint strong array, then there is some stage $s$ after which it never appears to. Hence for any finite set $F$, a copy of $\{n\} \oplus F$ appears in $\cup_{s} \mathcal{G}_{s}$.

Similarly, if $F$ is finite and $D_{\varphi_{n}(m)} \subseteq F$ for some $m$, then there is a stage $s$ such that $\varphi_{n, s}(m) \downarrow$ and $\max \{x \mid x \in F\}<s$. Thus the component of the form $\{n\} \oplus \sigma$ with $\sigma \subset \chi_{F}$ present in $\mathcal{G}_{s}$ will always be extended by 0 at future stages of the construction, and hence there is a component of the form $\{n\} \oplus F$ in $\cup_{s} \mathcal{G}_{s}$.

Suppose $\{n\} \oplus F \in \cup_{s} \mathcal{G}_{s}$, and $\varphi_{n}$ codes a disjoint strong array. Some $\{n\} \oplus \sigma$ with $\sigma \subset \chi_{F}$ was introduced into $\cup_{s} \mathcal{G}_{s}$. at some stage, and there must have been some later stage when $\varphi_{n}$ converged on one more number. At such a stage, we made the commitment to extend $\{n\} \oplus \sigma$ to $\left.\{n\} \oplus \chi_{(Y[k]}-\{0, \ldots, s\}\right) \cup F_{\sigma}$, where $k$ was such that if $\varphi_{n}$ is total then so is $\varphi_{k}$ and $\left\{\varphi_{k}(x)\right\}_{x \in \omega}=\left\{\varphi_{n}(x) \mid D_{\varphi_{n}(x)} \cap\{0, \ldots, s\}=\emptyset\right\}_{x \in \omega}$. Since $\varphi_{n}$ coded a disjoint strong array, so did $\varphi_{k}$. So $(\exists m)\left[D_{\varphi_{k}(m)} \subseteq Y^{[k]}\right]$. By definition of $\varphi_{k}$, there exists $l$ such that $\varphi_{k}(m)=\varphi_{n}(l)$, and $D_{\varphi_{n}(l)} \cap\{0, \ldots s\}=\emptyset$. Thus $D_{\varphi_{n}(l)} \subseteq Y^{[k]}-\{0, \ldots, s\}=F-\{0, \ldots, s\} \subseteq F$, as desired.

For this graph, we have a complete characterization that the degrees that can compute a copy are exactly the hyperimmune degrees.

Theorem 5.2 There exists an X-computable copy of $\mathcal{G}$ if and only if $X$ has hyperimmune degree.

Proof. Suppose $X$ has hyperimmune degree. Then there exists a function $g \leq_{T} X$ such that $g$ is not dominated by any computable function.

We construct $Y \leq_{T} X$ such that $(\forall e)\left[\left|Y^{[e]}\right|<\infty\right]$ and $(\forall e)\left[\left\{D_{\varphi_{e}(m)}\right\}_{m \in \omega}\right.$ is a disjoint strong array $\rightarrow$ $\left.(\exists m)\left[D_{\varphi_{e}(m)} \subseteq Y^{[e]}\right]\right]$, by finite extensions using $g$ as follows.

Let $\sigma_{s}^{[e]}$ denote the initial segment of the characteristic function of $Y^{[e]}$ that has been defined at the end of stage $s$.

Stage 0: Let $\sigma_{0}^{[e]}=\emptyset$.

Stage s+1: For all $e \leq s$, do as follows. If $\neg(\exists m)\left[D_{\varphi_{e, s+1}(m) \downarrow} \subseteq F_{Y_{s}^{[e]}}\right]$, then if $\varphi_{e, g(s)}(m) \downarrow$ for some $m \leq g(s)$ with $\min \left\{D_{\varphi_{e}(m)}\right\}>s$, let $\sigma_{s+1}^{[e]} \supset \sigma_{s}^{[e]}$ be such that $D_{\varphi_{e}(m)} \subseteq Y_{s+1}^{[e]}$. For all other $e \leq s$, let $\sigma_{s+1}^{[e]}=\sigma_{s}^{[e]} \gamma$. 
Note that if $\varphi_{e}$ codes a strong array, then the function $\psi_{e}(s)=\mu t \exists m\left[D_{\varphi_{e, t}(m) \downarrow} \geq s\right]$ is computable, and hence there are infinitely many $s$ where $g(s) \geq \psi_{e}(s)$. At the first stage $s$ such that $g(s) \geq \psi_{e}(s)$ we guarantee that $(\exists m)\left[D_{\varphi_{e}(m)} \subseteq Y^{[e]}\right]$.

The converse is as in Theorem 4.3. Suppose there is an $X$-computable copy of $\mathcal{G}$. Then there exists $Y \leq_{T} X$ such that $(\forall e)\left[\left|Y^{[e]}\right|<\infty\right]$ and $(\forall e)\left[\left\{D_{\varphi_{e}(m)}\right\}_{m \in \omega}\right.$ is a disjoint strong array $\left.\rightarrow(\exists m)\left[D_{\varphi_{e}(m)} \subseteq Y^{[e]}\right]\right]$. We will build a bi-hyperimmune set $Z \leq_{T} Y$. Since the hyperimmune degrees are upward closed, this will show that $X$ has hyperimmune degree.

We meet for all $e \in \omega$ the requirements:

$$
\begin{aligned}
& R_{e}:\left\{D_{\varphi_{e}(m)}\right\}_{m \in \omega} \text { is a disjoint strong array } \rightarrow(\exists m)\left[D_{\varphi_{e}(m)} \subseteq Z\right] \\
& S_{e}:\left\{D_{\varphi_{e}(m)}\right\}_{m \in \omega} \text { is a disjoint strong array } \rightarrow(\exists m)\left[D_{\varphi_{e}(m)} \subseteq \bar{Z}\right]
\end{aligned}
$$

Let $h(i, s)$ be a computable function of two variables such that if $\varphi_{i}$ is total then so is $\varphi_{h(i, s)}$ and $\left\{\varphi_{h(i, s)}(x)\right\}_{x \in \omega}=$ $\left\{\varphi_{i}(x) \mid D_{\varphi_{i}(x)} \cap\{0, \ldots, s\}=\emptyset\right\}_{x \in \omega}$.

The proof then follows exactly as in Theorem 4.3, where instead of defining $f_{s+1}(i)$ by letting $W_{f_{s+1}(i)}=$ $W_{i}-\{0, \ldots, s\}$, we let $f_{s+1}(i)=h(i, s+1)$, and similarly for $g$.

Corollary 5.3 For the graph $\mathcal{G}$ we have

$$
\mathbf{S p}(\mathcal{G})=\{\mathbf{x} \in \mathbf{D}: \mathbf{x} \text { is hyperimmune }\} .
$$

Hence, $\mathcal{G}$ is almost computable but the class of degrees $\mathbf{D}-\mathbf{S p}(\mathcal{G})$ is uncountable.

\section{At least bi-hyperhyperimmune}

Now consider

$$
\mathcal{H}=\left\{\{n\} \oplus F|n \in \omega \wedge| F \mid<\infty \wedge\left(\left\{W_{\varphi_{n}(m)}\right\}_{m \in \omega} \text { is a disjoint weak array } \rightarrow(\exists m)\left[W_{\varphi_{n}(m)} \subseteq F\right]\right)\right\}
$$

Lemma 6.1 If there exists an $X$-computable copy of $\mathcal{H}$ then there exists $Y \equiv_{T} X$ such that $(\forall e)\left|Y^{[e]}\right|<\infty$ and $(\forall e)\left[\left\{W_{\varphi_{e}(m)}\right\}_{m \in \omega}\right.$ is a disjoint weak array $\left.\rightarrow(\exists m)\left[W_{\varphi_{e}(m)} \subseteq Y^{[e]}\right]\right]$.

Proof. Suppose we have an $X$-computable copy of $\mathcal{H}$. To define $Y^{[e]}$, search for a component of the form $\{e\} \oplus F$ with $e \in X$ iff $0 \in F$, and let $Y^{[e]}=F$.

Lemma 6.2 For any partial computable function $\varphi_{n}$ and any finite set $D_{y}$, we can compute effectively from $n$ and $y$ an index $k$ such that if $\left\{W_{\varphi_{n}(m)}\right\}_{m \in \omega}$ is a disjoint weak array, then $\left\{W_{\varphi_{k}(m)}\right\}_{m \in \omega}$ is also a disjoint weak array, for all $m \in \omega W_{\varphi_{k}(m)} \cap D_{y}=\emptyset$, and for all $l \in \omega$ there exists $m \in \omega$ such that $W_{\varphi_{n}(m)} \subseteq W_{\varphi_{k}(l)}$.

Proof. Stage 0: Let $\alpha(m, 0)=m$ for all $m \in \omega$. Let $\beta(0)=1$.

Stage $s+1$ : For the least $m \leq s$ such that $W_{\varphi_{n}(\alpha(m, s))}[s+1] \cap D_{y} \neq \emptyset$, let $\alpha(m+l, s+1)=\beta(s)+l$ for all $l \in \omega$. Let $\alpha(r, s+1)=\alpha(r, s)$ for all $r<m$. Let $\beta(s+1)=\beta(s)+s+1$.

For all $x \leq s$, let $W_{\varphi_{k}(x), s+1}=W_{\varphi_{k}(x), s} \cup W_{\varphi_{n}(\alpha(x))}[s+1]$.

If $\left\{W_{\varphi_{n}(m)}\right\}_{m \in \omega}$ is a disjoint weak array, then since $D_{y}$ is finite, $\alpha(m)=\lim _{s} \alpha(m, s)$ exists and is finite for all $m$. Thus for all $m \in \omega, W_{\varphi_{n}(\alpha(m))} \subseteq W_{\varphi_{k}(m)}$. The array $\left\{W_{\varphi_{k}(m)}\right\}_{m \in \omega}$ is also a disjoint weak array because whenever we redefine $\alpha(m, s+1)$ we choose a new index that we haven't started copying yet.

Theorem 6.3 If there exists an $X$-computable copy of $\mathcal{H}$ then $X$ has bi-hyperhyperimmune degree. 
Pro of. To show that every $X$-computable copy of $\mathcal{H}$ computes a bi-hyperhyperimmune set is as in Theorems 4.3 and 5.2, using Lemma 6.2 to redefine the functions $f_{s}$ and $g_{s}$. However, we do not know whether the bi-hyperhyperimmune degrees are upward closed. Thus we will combine the method of Theorem 4.3 with a coding of $X$ back into the bi-hyperhyperimmune set that we build.

Suppose there is an $X$-computable copy of $\mathcal{F}$. Then there exists $Y \equiv_{T} X$ such that $(\forall e)[|Y[e]|<\infty]$ and $(\forall e)\left[\left\{W_{\varphi_{e}(m)}\right\}_{m \in \omega}\right.$ is a disjoint weak array $\left.\rightarrow(\exists m)\left[W_{\varphi_{e}(m)} \subseteq Y^{[e]}\right]\right]$. We will build a bi-hyperhyperimmune set $Z \leq_{T} Y$, and code $Y$ back into $Z$.

To ensure bi-hyperhyperimmunity, we meet for all $e \in \omega$ the requirements:

$$
\begin{aligned}
& R_{e}:\left\{W_{\varphi_{e}(m)}\right\}_{m \in \omega} \text { is a disjoint weak array } \rightarrow(\exists m)\left[W_{\varphi_{e}(m)} \subseteq Z\right] \\
& S_{e}:\left\{W_{\varphi_{e}(m)}\right\}_{m \in \omega} \text { is a disjoint weak array } \rightarrow(\exists m)\left[W_{\varphi_{e}(m)} \subseteq \bar{Z}\right]
\end{aligned}
$$

To code $Y$ into $Z$, we meet for all $e \in \omega$ the requirements:

$$
C_{e}: 0(01)^{e+3} 1 \text { is a substring of } \chi_{Z} \Longleftrightarrow e \in Y
$$

Moreover, for $m \in Y, n \in Y$, if $m<n$ then the string $0(01)^{m+3} 1$ will occur before the string $0(01)^{n+3} 1$ in $\chi_{Z}$.

In the construction, we will make use of functions $f_{s}(e)$ and $g_{s}(e)$ that keep track of the witness sets $Y^{\left[f_{s}(e)\right]}$ and $Y^{\left[g_{s}(e)\right]}$ that we are attempting to keep in or out of $Z$ to meet the requirements $R_{e}$ and $S_{e}$, respectively. For coding $Y$ into $Z$, first note that $Y$ is infinite. We will code the members of $Y$ into the characteristic function $\chi_{Z}$, in increasing order. We will use the function $h(s)$ to keep track of the next member of $Y$ that is to be coded into $\chi Z$.

Let $k(e, s)$ be a computable function such that if $\left\{W_{\varphi_{e}(m)}\right\}_{m \in \omega}$ is a disjoint weak array, then $\left\{W_{\varphi_{k(e, s)}(m)}\right\}_{m \in \omega}$ is also a disjoint weak array, for all $m \in \omega W_{\varphi_{k(e, s)}(m)} \cap\{0, \ldots, s\}=\emptyset$, and for all $l \in \omega$ there exists $m \in \omega$ such that $W_{\varphi_{e}(m)} \subseteq W_{\varphi_{k(e, s)}(l)}$. Such a function exists by Lemma 6.2.

Stage 0: Let $f_{0}(e)=g_{0}(e)=e$ for all $e \in \omega$. Let $h(0)=\mu y[y \in Y]$.

Stage $s+1$ : Choose the least $e \leq h(s)$ such that $\left[\left[s \in Y^{\left[f_{s}(e)\right]} \vee s+1 \in Y^{\left[f_{s}(e)\right]}\right] \wedge(\forall i<e)[s, s+1 \notin\right.$ $\left.\left.Y^{\left[f_{s}(i)\right]} \cup Y^{\left[g_{s}(i)\right]}\right]\right] \vee\left[\left[s \in Y^{\left[g_{s}(e)\right]} \vee s+1 \in Y^{\left[g_{s}(e)\right]}\right] \wedge(\forall i \leq e)\left[s, s+1 \notin Y^{\left[f_{s}(i)\right]}\right] \wedge(\forall i<e)\left[s, s+1 \notin Y^{\left[g_{s}(i)\right]}\right]\right]$. If $s \in Y^{\left[f_{s}(e)\right]}$ or $s+1 \in Y^{\left[f_{s}(e)\right]}$, then declare $s, s+1 \in Z$; for $i>e$ set $f_{s+2}(i)=k(i, s+1)$; and for $i \geq e$ set $g_{s+2}(i)=k(i, s+1)$. Move to stage $s+3$ of the construction. If $s \in Y^{\left[g_{s}(e)\right]}$ or $s+1 \in Y^{\left[g_{s}(e)\right]}$, then declare $s, s+1 \notin Z$; for $i>e$ set $f_{s+2}(i)=k(i, s+1)$; and for $i>e$ set $g_{s+2}(i)=k(i, s+1)$. Move to stage $s+3$ of the construction.

If no such $e$ exists, then we attempt to code $h(s)$ into $Z$. If for all $e \leq h(s), Y^{\left[f_{s}(e)\right]} \cap[s, s+2 h(s)+7]=\emptyset$ and $Y^{\left[g_{s}(e)\right]} \cap[s, s+2 h(s)+7]=\emptyset$, then extend the characteristic function of $Z$ by $0(01)^{h(s)} 1$; for $i>h(s)$ set $f_{s+2 h(s)+7}(i)=k(i, s+2 h(s)+7)$; for $i>e$ set $g_{s+2 h(s)+7}(i)=k(i, s+2 h(s)+7)$; set $h(s+2 h(s)+7)=$ $\mu y[y>h(s) \wedge y \in Y]$. Move to stage $s+2 h(s)+8$ of the construction.

Otherwise, declare $s, s+1 \in Z$ and leave $f_{s+2}=f_{s}, g_{s+2}=g_{s}, h(s+2)=h(s)$, and move to stage $s+3$ of the construction.

Verification: The construction worked by having a global requirement that for $n \notin Y, 0(01)^{n+3} 1$ is not a substring of $\chi_{Z}$, and by meeting the other requirements in the priority order $R_{e}, S_{e}, C_{e}$.

Lemma 6.4 For $n \notin Y, 0(01)^{n+3} 1$ is not a substring of $\chi_{Z}$.

Proof. Towards meeting requirements for $R_{e}$ and $S_{e}$, the characteristic function of $Z$ is always extended by pairs, 00 or 11, since when taking action for such a requirement at a stage $s+1$, either both $s$ and $s+1$ are enumerated into $Z$, or neither is. Hence working towards meeting $R_{e}$ or $S_{e}$ cannot result in the introduction of a string of the form $0(01)^{n+3} 1$. When coding in some $e \in Y$, we ensure that we introduce the entire string $0(01)^{e+3} 1$ at once, which is not an extension or substring of $0(01)^{n+3} 1$ for any $n \neq e$.

Lemma 6.5 For all e, $f(e)=\lim _{s} f_{s}(e)$ exists and $Y^{[f(e)]} \subseteq Z, g(e)=\lim _{s} g_{s}(e)$ exists and $Y^{[g(e)]} \subseteq \bar{Z}$, and $(\exists s)[h(s)>e]$. 
Pro of. Suppose the lemma holds for all $i<e$.

Let $s_{0}$ be a stage by which, for all $i<e, f_{s_{0}}(i)=f(i), g_{s_{0}}(i)=g(i), h\left(s_{0}\right)>i, s_{0}>\max \left\{Y^{[f(i)]}\right\}$, and $s_{0}>\max \left\{Y^{[g(i)]}\right\}$.

Since $h$ is a non-decreasing function and $e \leq h\left(s_{0}\right)$, we have $e \leq h(t)$ for all $t \geq s_{0}$. Thus it is easy to see from the construction, that for any $t \geq s_{0}$, if $t \in Y^{\left[f_{t}(e)\right]}$ then $t \in Z$. It is also easy to see that $f(e)=$ $\lim _{s} f_{s}(e)=f_{s_{0}}(e)$. Hence $Y^{[f(e)]} \subseteq Z$.

Let $s_{1}>s_{0}$ be such that $s_{1}>\max \left\{Y^{[f(e)]}\right\}$. Then, again, it is easy to see from the construction that $g(e)=g_{s_{1}}(e)$ and that $Y^{[g(e)]} \subseteq \bar{Z}$.

Let $s_{2}>s_{1}$ be such that $s_{2}>\max \left\{Y^{[g(e)]}\right\}$, and such that we visit stage $s_{2}$ of the construction. If $h\left(s_{2}\right)>e$, then we are done, so suppose $h\left(s_{2}\right)=e$. Then at stage $s_{2}$ of the construction we will code $e$ into the characteristic function of $Z$, and set $h\left(s_{2}+2 h\left(s_{2}\right)+7\right)>e$.

Since the construction is $Y$-computable, we have $Z \leq_{T} Y$. By Lemma 6.5, for each $e, Y^{[f(e)]} \subseteq Z$. Let $s$ be such that $f(e)=k(e, s)$. Then if $\left\{W_{\varphi_{e}(m)}\right\}_{m \in \omega}$ is a disjoint weak array, then $\left\{W_{\varphi_{k(e, s)}(m)}\right\}_{m \in \omega}$ is also a disjoint weak array. Hence there exists $l$ such that $W_{\varphi_{k(e, s)(l)}} \subseteq Y^{[k(e, s)]}=Y^{[f(e)]} \subseteq Z$. So there exists $m \in \omega$ such that $W_{\varphi_{e}(m)} \subseteq W_{\varphi_{k(e, s)}(l)} \subseteq Z$. Similarly, since $Y^{[g(e)]} \subseteq \bar{Z}$, if $\left\{W_{\varphi_{e}(m)}\right\}_{m \in \omega}$ is a disjoint weak array, then there exists $m \in \omega$ such that $W_{\varphi_{e}(m)} \subseteq \bar{Z}$. Hence $Z$ is bi-hyperhyperimmune. To see that $Y \leq_{T} Z$, recall that we've shown that the characteristic function of $Z$ contains a string of the form $0(01)^{n+3} 1$ if and only if $n \in Y$, and that these strings occur in order as $n$ increases. Hence, to $Z$-compute whether $n \in Y$, we search for the first string of the form $0(01)^{m+3} 1$ in the characteristic function of $Z$ with $m \geq n$. Then $n \in Y$ iff $m=n$.

Question 6.6 Is $\mathbf{S p}(\mathcal{H})$ exactly the bi-hyperhyperimmune degrees?

\section{Summary}

The bi-noncomputable degrees are exactly the noncomputable degrees. Wehner's example constructs a structure whose degree spectrum is exactly the (bi)-noncomputable degrees.

The bi-hyperimmune degrees are exactly the hyperimmune degrees. We have an example of a structure with degree spectrum exactly the (bi)-hyperimmune degrees.

The bi-immune degrees are a proper subset of the immune degrees. We have a candidate whose degree spectrum might be the bi-immune degrees, but we do not know.

The bi-hyperhyperimmune degrees are a proper subset of the hyperhyperimmune degrees. We have a candidate whose degree spectrum might be the bi-hyperhyperimmune degrees, but we don't even know if the bihyperhyperimmune degrees are upward closed.

Does there exist a structure the degree spectrum of which is exactly the hyperhyperimmune degrees? The "obvious" method that we tried to use to force hyperhyperimmunity actually did more and forced bi-hyperhyperimmunity. Of course, we are able to realize exactly the immune degrees as a degree spectrum, since these are just the noncomputable degrees, even though the "obvious" method of trying to force immunity actually forces bi-immunity.

\section{Question}

We close with a question about the possible degree spectra of linear orderings. Miller [16] constructed a linear order whose degree spectrum when restricted to the $\Delta_{2}^{0}$ degrees coincides exactly with the noncomputable $\Delta_{2}^{0}$ degrees. Later Downey and independently Chisholm noticed and communicated to Miller that the linear ordering Miller constructed is computable in every hyperimmune degree. Now it is natural to ask whether Miller's linear order (or indeed any other linear order) realizes any of the following spectra:

1. the immune degrees (all noncomputable degrees),

2. the bi-immune degrees, 
3. the hyperimmune degrees?

The first case would give an affirmative answer to Downey's question [3] on the existence of a noncomputable linear ordering which is computable in every noncomputable degree.

\section{References}

[1] S. Barry Cooper. Computability theory. Chapman \& Hall/CRC, Boca Raton, FL, 2004.

[2] Natasha L. Dobrinen and Stephen G. Simpson. Almost everywhere domination. J. Symbolic Logic, 69(3):914-922, 2004.

[3] Rodney G. Downey. On presentations of algebraic structures. Sorbi, Andrea (ed.), Complexity, logic, and recursion theory. New York, NY: Marcel Dekker. Lect. Notes Pure Appl. Math., 187:157-205, 1997.

[4] Sergey S. Goncharov, Valentina S. Harizanov, Julia F. Knight, Charles McCoy, Russell G. Miller and Reed Solomon. Enumerations in computable structure theory. Annals of Pure and Applied Logic, 136(3): 219-246, 2005.

[5] Valentina S. Harizanov. Pure computable model theory. In Handbook of recursive mathematics, Vol. 1, volume 138 of Stud. Logic Found. Math., pages 3-114. North-Holland, Amsterdam, 1998.

[6] Carl G. Jockusch, Jr. The degrees of bi-immune sets. Z. Math. Logik Grundlagen Math., 15:135-140, 1969.

[7] Carl G. Jockusch, Jr. Upward closure of bi-immune degrees. Z. Math. Logik Grundlagen Math., 18:285-287, 1972.

[8] Carl G. Jockusch, Jr. Upward closure and cohesive degrees. Israel J. Math., 15:332-335, 1973.

[9] Carl G. Jockusch, Jr. and Robert I. Soare. $\Pi_{1}^{0}$ classes and degrees of theories. Trans. Amer. Math. Soc., 173:33-56, 1972.

[10] Iskander Sh. Kalimullin. Spectra of degrees of some structures. Algebra Logika, 46(6):729-744, 2007.

[11] Iskander Sh. Kalimullin. Almost computably enumerable families of sets. Sb. Math., 199(10):1451-1458, 2008.

[12] Iskander Sh. Kalimullin. Almost computably enumerable families of sets. Siberian Math., 49(6):1034-1043, 2008.

[13] Julia F. Knight. Degrees coded in jumps of orderings. J. Symbolic Logic, 51(4):1034-1042, 1986.

[14] Stuart A. Kurtz. Notions of weak genericity. J. Symbolic Logic, 48:764-770, 1983.

[15] Juri T. Medvedev. On non-isomorphic recursively enumerable sets. Dokl. Akad. Nauk SSSR, 102:211-214, 1955.

[16] Russell Miller. The $\Delta_{2}^{0}$-spectrum of a linear order. J. Symbolic Logic, 66(2):470-486, 2001.

[17] Theodore A. Slaman. Relative to any nonrecursive set. Proc. Amer. Math. Soc., 126(7):2117-2122, 1998.

[18] Robert I. Soare. Recursively enumerable sets and degrees. Perspectives in Mathematical Logic. Springer-Verlag, Berlin, 1987. A study of computable functions and computably generated sets.

[19] Vladimir A. Uspenskij. Some notes on recursively enumerable sets. Z. Math. Logik Grundlagen Math., 3:157-170, 1957.

[20] Stephan Wehner. Enumerations, countable structures and Turing degrees. Proc. Amer. Math. Soc., 126(7):2131-2139, 1998. 